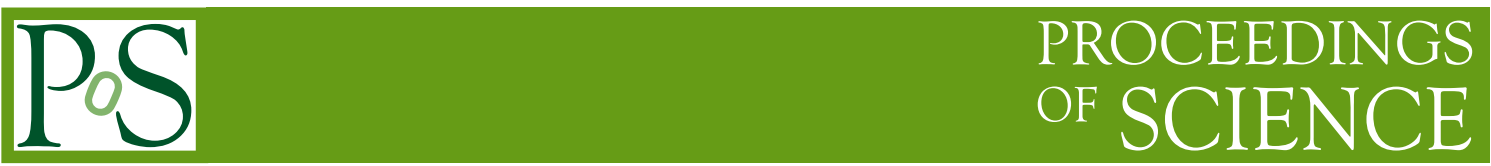

\title{
Condensation of dust in supernova ejecta
}

\section{Arkaprabha Sarangi \& Isabelle Cherchneff}

University of Basel, Switzerland

E-mail: arkaprabha.sarangi@unibas.ch

\begin{abstract}
Observations in the infrared and submm indicate the presence of molecules and dust in the ejecta of type II-P supernovae. The mass of dust formed in the ejecta of supernovae is still uncertain and highly debated: Infrared observations indicate smaller dust masses $\left(10^{-5}\right.$ to $\left.10^{-3} M_{\odot}\right)$ before 500 days post-explosion, compared to submm observations with Herschel revealing supernova remnants as large reservoirs of cool dust $\left(10^{-2}\right.$ to $\left.0.7 M_{\odot}\right)$. We study the ejecta of a typical type II-P supernova with a chemical kinetic approach considering a $15 M_{\odot}$ progenitor as a benchmark. The synthesis of molecules (e.g., $\mathrm{CO}, \mathrm{SiO}, \mathrm{O} 2, \mathrm{AlO}, \mathrm{SiS}, \mathrm{FeS}, \mathrm{SiC}, \mathrm{SO}$ ) and small clusters (e.g., silicates, carbon, metal oxides, metallic clusters etc.) in the gas phase is considered. The clusters form gradually over time in different ejecta zones, small dust masses form in the first 600 days $\left(\sim 10^{-4} M_{\odot}\right)$, that gradually increase up to $\sim 0.1 M_{\odot}$ at 1500 days post-explosion. The small clusters condense to form dust grains in the gas phase. The size distributions of different dust components are derived from the study.
\end{abstract}

The Life Cycle of Dust in the Universe: Observations, Theory, and Laboratory Experiments 18-22 November, 2013

Taipei, Taiwan 


\section{Introduction}

Evolved circumstellar environments are the most suitable loci for the synthesis of cosmic dust. The ejecta of a typical type II supernova provides physical conditions conductive to the synthesis of dust. Evidences of warm dust have been reported at infrared in supernovae (SN) ejecta around 300 days after explosion [1, 2, 3]. Recent observations with the Herschel telescope also indicates the presence of cool dust in the ejecta [4]. We model the ejecta of type II-P supernovae through chemical kinetics [5]. The formation of dust grains is preceded by the gas phase molecular synthesis and nucleation phase. The nucleation phase terminates at the formation of energetically stable small clusters.

\section{Grain condensation model}

The small clusters in the gas phase [5] simultaneously condense to form dust grains. The sizes of the stable small clusters produced as an end product of gas phase chemistry are given in table 1. The clusters grow through coagulation and coalescence in the free molecular regime to produce larger grains of individual dust species. The concentration of particles at different sizes are determined by solving the integro-differetial coagulation equation [6]. The rate of coagulation between grains is determined by the combined effect of Brownian diffusion, gravitational collection, turbulent inertial motion and shear, the Van der Waal's and the viscous forces. However, Brownian diffusion and Van der Waal's forces are the most effective processes in the free molecular regime. A semi-implicit volume conserved model has been developed to solve to the coagulation equation. The grains are assumed to maintain a spherical morphology and compact structure (particle density is equal to the bulk density). The grains of individual dust species are assigned to discrete bins following a volume ratio distribution given by $v_{n}=\gamma^{n-1} v_{0}$, where $v_{n}$ is the volume of the $n^{\text {th }}$ bin and $\gamma$ is a constant defined as the ratio of the volumes of adjacent bins. Particles with volumes intermediate to any two consecutive bins are allocated through a volume fractionation formalism demonstrated by [6]. The effect of coalescence between the particles are estimated through Van der Waal's forces following the study by [7].

\section{Grain size distribution}

Forsterite, alumina and amorphous carbon are the most abundant types of dust in the ejecta. Synthesis of forsterite and alumina occurs essentially in the $\mathrm{O} / \mathrm{Ne} / \mathrm{Mg}$ rich zones. Carbon dust is synthesized in the $\mathrm{He} / \mathrm{C}$ zone of the ejecta [5]. Forsterite and alumina grains are found to effectively condense between 650 to 750 days post-explosion. The typical ejecta temperature at this epoch ranges between 900 to $1500 \mathrm{~K}$. The largest grains of forsterite are of the order of $500 \AA$ and the distribution peaks around $200 \AA$ (Fig 1a). For alumina largest grains synthesized in the ejecta are around $400 \AA$ and the peak of the distribution lies at $150 \AA$ (Fig 1b). Due to the presence of $\mathrm{He}^{+}$ in the ejecta, carbon dust starts forming in the ejecta as late as 1000 days post-explosion. Owing to the low gas densities at late times, carbon dust cannot grow very large grains even though it is more abundant than other dust components in the ejecta. The peak of the distribution is roughly at $200 \AA$ (Fig 1c) and a fraction of the total carbon remains in the form of grains smaller than $50 \AA$. 

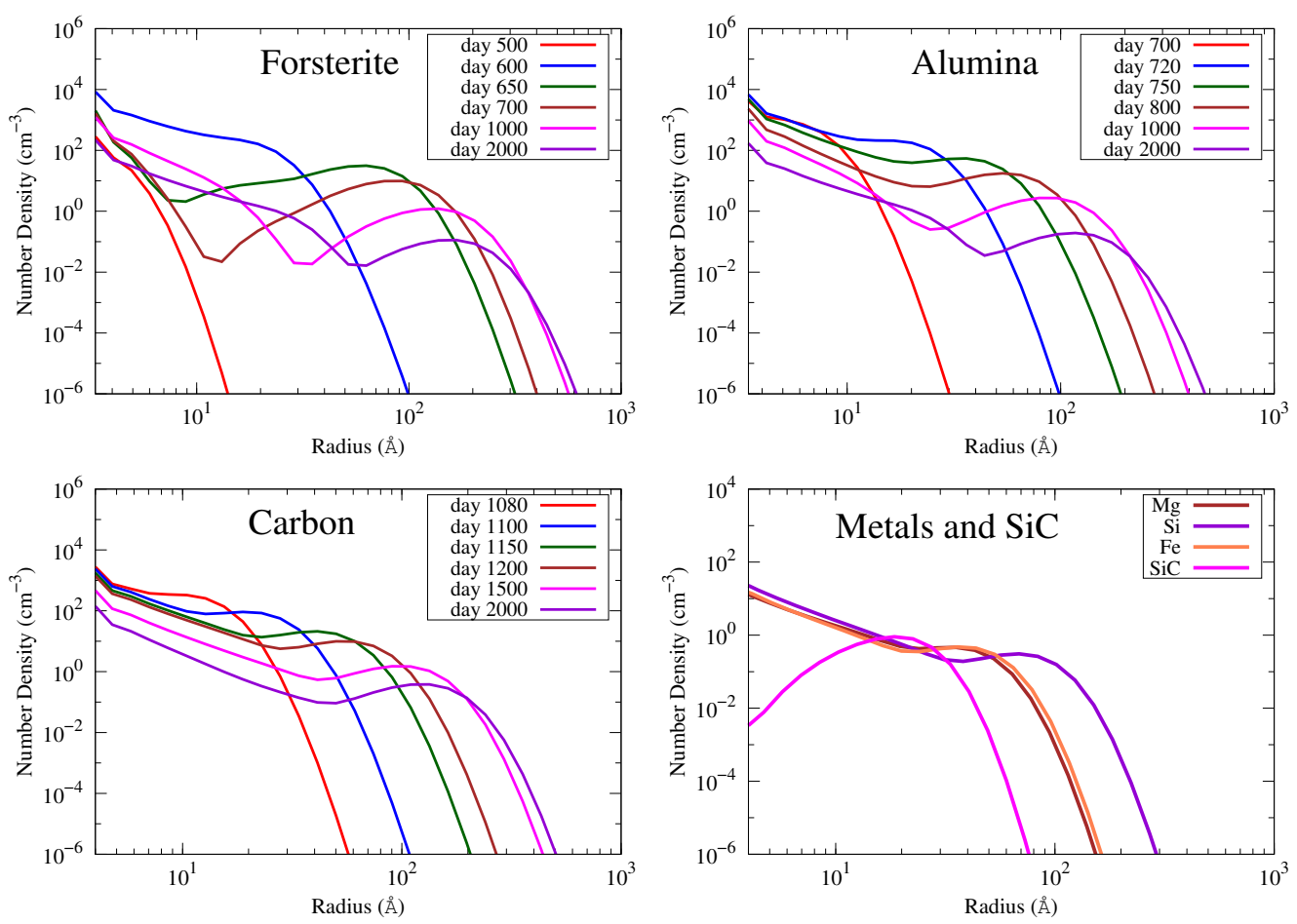

Figure 1: Grain size distributions at different epochs are plotted as a function of particle sizes for the three prime components: Forsterite (top-left, 1a), Alumina (top-right, 1b) and Carbon (bottomleft, 1c). The grain size distribution profiles at 2000 day post-explosion, are presented for the metallic grains and silicon carbide (bottom-right, 1d).

Table 1: The radii $(\AA)$ of the stable clusters produced as an end product in the gas phase

\begin{tabular}{ccccccc}
\hline$\left(\mathrm{Mg}_{2} \mathrm{SiO}_{4}\right)_{2}$ & $\left(\mathrm{Al}_{2} \mathrm{O}_{3}\right)_{4}$ & $\mathrm{C}_{28}$ & $(\mathrm{SiC})_{4}$ & $(\mathrm{Si})_{4}$ & $(\mathrm{Fe})_{4}$ & $(\mathrm{Mg})_{4}$ \\
\hline 3.33 & 3.45 & 3.93 & 2.15 & 2.45 & 2.81 & 2.29 \\
\hline
\end{tabular}

Apart from the major dust components, relatively less abundant species of dust as silicon carbide, metallic sulphur, iron, and magnesium are also present in the ejecta. Silicon carbide is synthesized in the $\mathrm{He} / \mathrm{C}$ zone, whereas metallic sulphur and iron grains form in the innermost $\mathrm{Si} / \mathrm{S}$ zone [5]. The residual magnesium in the O-core, after the formation of magnesium-silicates, tends to form pure metallic clusters. The peak of the size distribution profile for the small clusters lies between 20 to $100 \AA$ at 2000 days post-explosion (Fig 1d). The chances of survival of these small grains after the passage of the reverse shock through the ejecta are in question.

\section{Total budget of dust}

The small clusters synthesized in the gas phase efficiently condense to form dust grains in the ejecta. Thus the total mass of small clusters in the gas phase is the upper limit of the total dust mass in SNe ejecta [5]. The total mass of dust in the ejecta considering grains larger than $50 \AA$ is presented in Figure 5 in solar units. Forsterite starts to form as early as 300 days post-explosion and grows from $10^{-6} M_{\odot}$ at 400 days to $5 \times 10^{-3} M_{\odot}$ at 700 days. Alumina grains starts to appear 


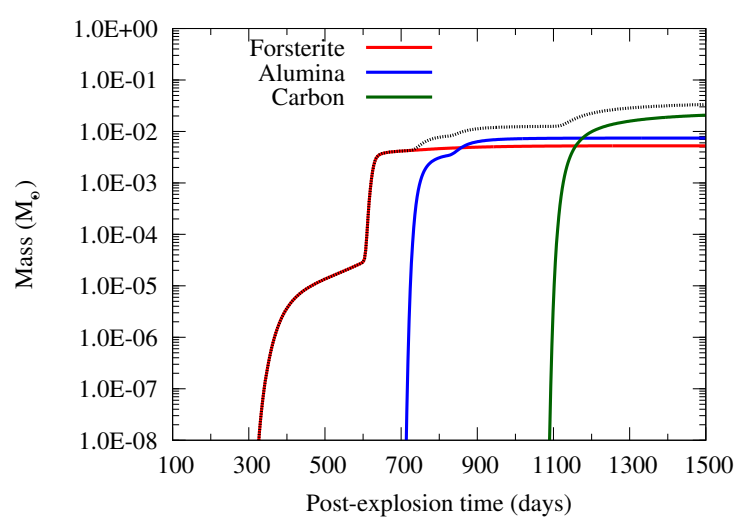

Figure 2: Total dust budget of major dust species in the ejecta considering grains larger than $50 \AA$

around 700 days are grows rapidly to $10^{-2} M_{\odot}$ by 900 days. Carbon dust forms at late times and grows to $2 \times 10^{-2} M_{\odot}$ by 1500 days post explosion. The trend of gradual increase in dust mass and the final mass of condensed dust in the ejecta agree well with the mass of dust observed at infrared and submm wavelengths.

\section{Discussion}

The formalism developed in this study couples the gas phase chemistry to the condensation of grains. The model considers the synthesis of dust grains in the ejecta of type II-P supernovae in a dynamic environment where non-equilibrium chemistry prevails. New grain size distributions are obtained which are dependent on the type of dust species and ejecta conditions. This provides the necessary tool to analyse the fluxes in SNe ejecta. It also provides the necessary inputs to study the effect of the passage of a reverse shock through the SNe remnants.

\section{References}

[1] R. Kotak et al. Early-time SPITZER observations of the type II plateau supernova SN 2004dj 2005 ApJL, 628, L123

[2] P. F. Roche et al. Silicon monoxide in 1987A 1991 MNRAS, 252, 39

[3] J. Spyromilio et al. Carbon monoxide in supernova SN1987A 1988 Nature, 334, 327

[4] M. Matsuura, E. Dwek, M. Meixner et al. Herschel detects a massive dust reservoir in supernova SN1987A 2011 Sci, 333, 1258

[5] A. Sarangi, I. Cherchneff The chemically controlled synthesis of dust in type II-P supernovae 2013 ApJ, 776, 107

[6] M. Jacobson Fundamentals of atmospheric modeling 2005 Cambridge University Press

[7] M. G. Sceats Brownian coagulation in aerosols - the role of long range forces 1988 J. Collid. Sci vol. 129 